\title{
Stretch induced hyperexcitability of mice callosal pathway
}

\author{
Anthony Fan ${ }^{1}$, Kevin A. Stebbings ${ }^{2}$, Daniel A. Llano ${ }^{2,3,4,5}$ and Taher Saif ${ }^{1 *}$ \\ ${ }^{1}$ Department of Mechanical Science and Engineering, University of Illinois, Urbana, IL, USA, ${ }^{2}$ Neuroscience Program, \\ University of Illinois, Urbana, IL, USA, ${ }^{3}$ Department of Molecular and Integrative Physiology, University of Illinois, Urbana, IL, \\ USA, ${ }^{4}$ Beckman Institute, University of Illinois, Urbana, IL, USA, ${ }^{5}$ College of Medicine, University of Illinois, Urbana, IL, USA
}

Memory and learning are thought to result from changes in synaptic strength. Previous studies on synaptic physiology in brain slices have traditionally been focused on biochemical processes. Here, we demonstrate with experiments on mouse brain slices that central nervous system plasticity is also sensitive to mechanical stretch. This is important, given the host of clinical conditions involving changes in mechanical tension on the brain, and the normal role that mechanical tension plays in brain development. A novel platform is developed to investigate neural responses to mechanical stretching. Flavoprotein autofluoresence (FA) imaging was employed for measuring neural activity. We observed that synaptic excitability substantially increases after a small (2.5\%) stretch was held for $10 \mathrm{~min}$ and released. The increase is accumulative, i.e., multiple stretch cycles further increase the excitability. We also developed analytical tools to quantify the spatial spread and response strength. Results show that the spatial spread is less stable in slices undergoing the stretch-unstretch cycle. FA amplitude and activation rate decrease as excitability increases in stretch cases but not in electrically enhanced cases. These results collectively demonstrate that a small stretch in physiological range can modulate neural activities significantly, suggesting that mechanical events can be employed as a novel tool for the modulation of neural plasticity.

Katsuei Shibuki,

Niigata University, Japan Bryan Pfister, New Jersey Institute of Technology,

Keywords: neuromechanics, acute brain slice, hyperexcitability, plasticity, mechanical stretch, flavoproteins autofluorescence

USA

*Correspondence: Taher Saif,

Mechanical Science and Engineering, University of Illinois, 1206 W. Green St., Urbana, IL 61801, USA saif@illinois.edu

Received: 30 April 2015 Accepted: 15 July 2015 Published: 05 August 2015

Citation:

Fan A, Stebbings KA, Llano DA and Saif $T$ (2015) Stretch induced hyperexcitability of mice callosal pathway. Front. Cell. Neurosci. 9:292. doi: 10.3389/fncel.2015.00292

\section{Introduction}

Modulation of neuronal communications has traditionally been viewed as chemically induced (Kandel et al., 2012). However, recent evidence shows that mechanical cues influence many biological processes (Wang et al., 2009). Neurons are particularly sensitive to their mechanical micro-environment. For example, ion channels are mechano-sensitive, such that a high-enough stress induces structural changes in a protein complex, and hence a change in functionality (Sachs, 2010). This has been shown experimentally in a variety of systems (Pfister et al., 2006; Cohen et al., 2007; Morris, 2011). In addition, growth of neurons can be induced by an applied stretch (Pfister et al., 2004). For example, dorsal root ganglion neurons are able to respond to a steady stretch and adjust their length to as far as a thousand times their original length. Application of stretch in the physiological range to frog neuromuscular junctions increases the frequency of spontaneous activities and the amplitude of evoked activities (Chen and Grinnell, 1995) within tens of milliseconds. Embryonic drosophila motor neurons actively maintain a rest tension of about a nano-Newton, and the loss of that tension prevents neurotransmitter vesicle clustering at the 
presynaptic terminal (Siechen et al., 2009). Finally, stretching the axon of the motor neurons by $5 \%$ for $30 \mathrm{~min}$ increases vesicle clustering by $200 \%$ (Siechen et al., 2009).

Central nervous system neurons are also subjected to large mechanical stretches and tension with varying rates during development, tumor growth and injuries, and brain swelling. It has been shown that tension is able to direct the growth of primary hippocampal neuron in 2-D culture (Lamoureux et al., 2002). CNS axons can also be stretch grown to $1 \mathrm{~cm}$ in length (Smith et al., 2001). Various groups have looked at the effect of traumatic strain, strain rate, and stress on cell death regulatory mechanism (Morrison et al., 2011; Franze et al., 2013) in animal models (Santhakumar et al., 2001), dissociated primary culture (Patel et al., 2014), and cortical cell culture (Goforth et al., 2011; Ferrario et al., 2013). However, the effects of mechanical stretches at the physiological level on long and short term synaptic functions, in particular, neuronal excitability remain unclear.

Although it has been speculated that mechanical forces at the physiological level play a role in brain morphology (Van Essen, 1997) and function (Tyler, 2012), no direct observations have yet been made to demonstrate their effect on brain functionality. In this study, we show, using untreated mouse brain coronal slices, that the history of both a small stretch and a small stretch rate can substantially modulate evoked and spontaneous neural activities in the callosal pathway. We chose the coronal preparation of the callosal axon pathway for its strong axon projection bridging the 2 hemispheres. The pathway consists of a large number of axonal projections. Thus, the preparation is appropriate for testing the hypothesis that mechanical stretch along the pathway will affect its function, in particular its excitability. We developed an experimental setup that allows us to apply a prescribed amount of stretch in one direction across the brain slice, modeling an increased physiological tension along the callosal axonal projections. Concurrently, the setup allows evaluation of spontaneous and electrically evoked neural activities. These activities are quantified by imaging flavoprotein autofluorescence (FA), which originates from the change of oxidation state of mitochondrial flavoproteins upon neuronal activation (Shibuki et al., 2003; Reinert et al., 2007).

\section{Materials and Methods}

\section{Brain Slicing}

One month old mice of both sexes were obtained from the in-house animal facility at Beckman Institute for Advanced Science and Technology at the University of Illinois. The animal was deeply anesthetized with ketamine $(100 \mathrm{mg} / \mathrm{kg})$ and xylazine $(3 \mathrm{mg} / \mathrm{kg})$ and a transcardial perfusion was subsequently performed with chilled oxygenated (95\% O2/5\% $\mathrm{CO} 2)$ slicing solution [sucrose $(234 \mathrm{mM})$, glucose $(11 \mathrm{mM})$, $\mathrm{NaHCO} 3(26 \mathrm{mM}), \mathrm{KCl}(2.5 \mathrm{mM}), \mathrm{NaH} 2 \mathrm{PO} 4 \bullet \mathrm{H} 2 \mathrm{O}(125 \mathrm{mM})$, $\mathrm{MgCl} 2 \bullet 6 \mathrm{H} 2 \mathrm{O}(10 \mathrm{mM})$, and $\mathrm{CaCl} 2 \bullet 2 \mathrm{H} 2 \mathrm{O}(0.5 \mathrm{mM})]$ at $4^{\circ} \mathrm{C}$. The mouse was then decapitated and its brain was removed from the skull. The aligned brain tissue was submerged in chilled slicing fluid in a vibration slicer. Obtained slices $600 \mu \mathrm{m}$ thick were left in a warm $\left(32^{\circ} \mathrm{C}\right)$ artificial cerebrospinal fluid (aCSF)
[NaHCO3 $(26 \mathrm{mM}), \mathrm{KCl}(2.5 \mathrm{mM})$, glucose $(10 \mathrm{mM}), \mathrm{NaCl}$ $(126 \mathrm{mM}), \mathrm{NaH} 2 \mathrm{PO} 4 \bullet \mathrm{H} 2 \mathrm{O}(1.25 \mathrm{mM}), \mathrm{MgCl} 2 \bullet 6 \mathrm{H} 2 \mathrm{O}(3 \mathrm{mM})$, and $\mathrm{CaCl} 22 \mathrm{H} 2 \mathrm{O}(1.1 \mathrm{mM})]$ bath for an hour before experiments. We have previously found that $600 \mu \mathrm{m}$ thick slices prepared in a similar fashion are robustly viable (Llano et al., 2014). In this study, all data are obtained from the callosal pathway. All procedures were approved by the Institutional Animal Care and Use Committee at the University of Illinois. All animals were housed in animal care facilities approved by the American Association for Accreditation of Laboratory Animal Care. Every attempt was made to minimize the number of animals used and to reduce suffering at all stages of the study.

\section{Stretching Mechanism}

Slices were gripped and made stationary by a micro-vice on each side of the perfusion stage as depicted in Figures 1A-C. This was intended to avoid the use of an adherent stretchable substrate leading to a mismatch in elastic properties. Each microvice is connected to the frame of the recording chamber. A power screw mechanism controlled by a stepper motor is used to actuate the micro-vices toward or away from each other. Each clockwise turn of the screw would lead to a linear retraction of $1 / 80$ inches and vice versa, hence stretching/relaxing the mounted slice. Geometries were optimized so that the whole device could be fit under the fluorescence microscope used for imaging during the experiment. The inlet and outlet attached to the recording chamber enable aCSF [NaHCO3 $(26 \mathrm{mM}), \mathrm{KCl}$ $(2.5 \mathrm{mM})$, glucose $(10 \mathrm{mM}), \mathrm{NaCl}(126 \mathrm{mM}), \mathrm{NaH} 2 \mathrm{PO} 4 \bullet \mathrm{H} 2 \mathrm{O}$ $(1.25 \mathrm{mM}), \mathrm{MgCl} 2 \bullet 6 \mathrm{H} 2 \mathrm{O}(2 \mathrm{mM})$, and $\mathrm{CaCl} 2 \bullet 2 \mathrm{H} 2 \mathrm{O}(2 \mathrm{mM})]$ to perfuse the slice at a constant flow rate with no apparent waste accumulation.

\section{Flavoprotein Autofluorescence Imaging}

FA was used in this study to track neuronal activities. Briefly, oxidation of mitochondrial flavoproteins is induced by influx of calcium ions upon neuronal activation (Shibuki et al., 2003). The oxidized flavoproteins fluoresce green under blue light. By tracking this green autofluorescence, neural activity could be measured. We and others have shown that it is a highly sensitive marker of neuronal activation and is quantitative (Reinert et al., 2007; Llano et al., 2009, 2012). Importantly, and unlike other imaging methods, the FA imaging signals remain stable for hours in a slice preparation (Shibuki et al., 2003). This permits a series of manipulations to be done in the tissue to investigate potential mechanisms by which micro-stretch affects neuronal activity. It has been shown that $80 \%$ of the FA signal comes from postsynaptic activations (Reinert et al., 2004; Llano et al., 2009). The method provides a rapid assessment of neuronal activation and carries several advantages compared to other imaging techniques that involve dye loading. Dye loading (e.g., calcium-sensitive or voltage-sensitive dyes) creates substantial changes in signal strength over time and potential heterogeneities over the tissue, depending on dye uptake (Lajtha et al., 2007). Electrophysiological measurements are of limited use in the current experiments because upon the induction of stretch, slippage might occur between the electrode-tissue interface. In this study, the slice was put under an epifluorescence 

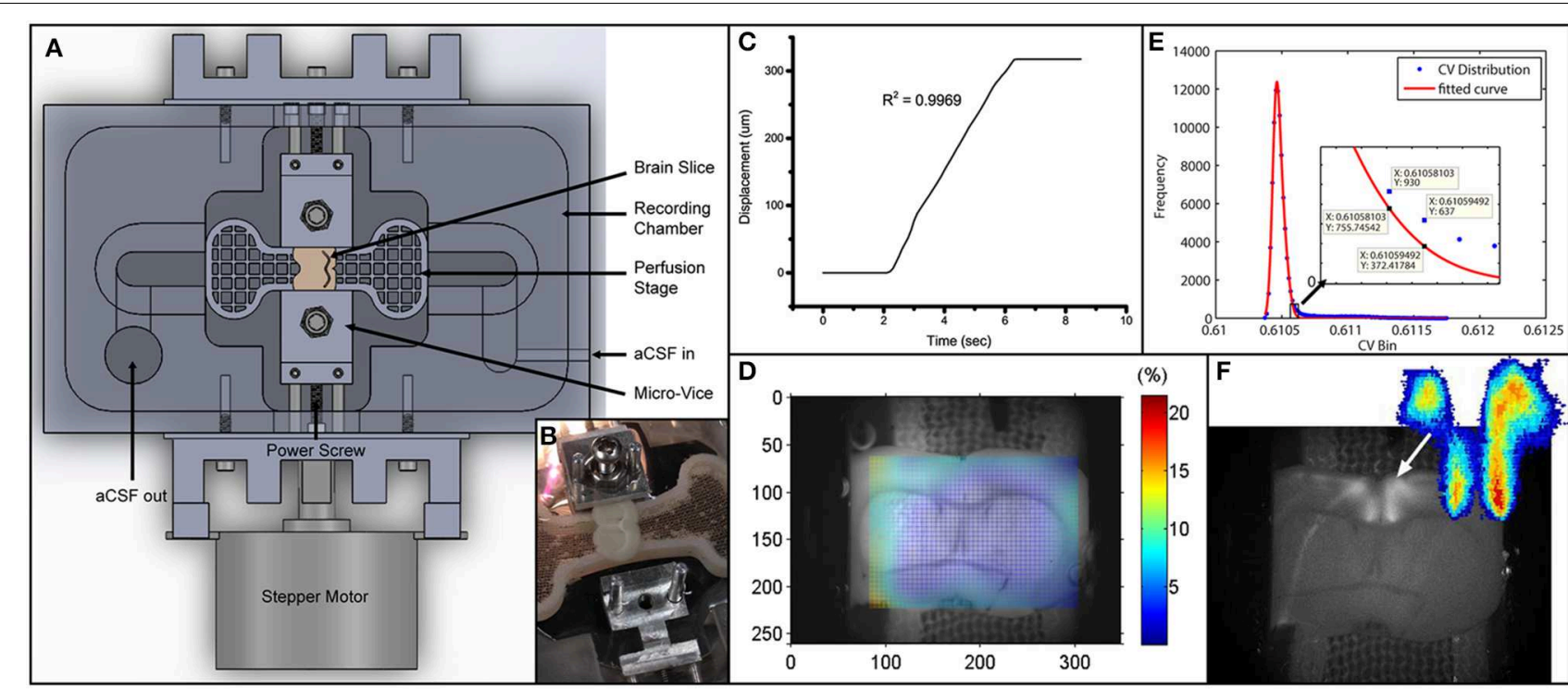

FIGURE 1 | Experimental setup and data analysis methods. (A) Schematics depicting the entire setup. Micro-vices are actuated by a power screw mechanism in which a rotation of the screw will result in a uniaxial translation. (B) Picture of device with brain slice secured on one end. (C) Displacement vs. time plot depicting the stretch applied during the experiment. $R^{2}$-value describes a linear fit to the increasing region. The displacement is $317.5 \mu \mathrm{m}$ over $4 \mathrm{~s}$, giving a stretch rate of $79.4 \mu \mathrm{m} / \mathrm{s}$. (D) Digital image correlation (see Materials and Methods) was used to obtain displacement fields from videos. The color map here plots the effective strain (Equation 2) over the brain slice preparation. (E) $I_{C V}$ of all pixels are plotted as a histogram in blue circles. Red line depicts a 2-term Gaussian fit. The right hand side is magnified in the subplot. Threshold value based on Equation (3) is sandwiched between the 2 data labels. (F) Grayscale image was obtained by performing a standard deviation projection in ImageJ. The heat map was generated by plotting $\mathrm{I}_{\mathrm{CV}}^{\mathrm{p}}$. Sparse outliers away from the main area were also trimmed. microscope [Olympus BX51 with a Prior Lumen 200 light source] using an UMNIB Olympus filter cube (470-490-nmexcitation, 505-nm dichroic, and 515-nm emission long pass) and other supplementary optics (Theyel et al., 2011). Images were acquired with a CCD camera [QImaging EXi with Firewire interface] at 4 frames per second. Image analyses were done in ImageJ and MATLAB.

\section{Electrical and Chemical Stimulation}

In all experiments, $800 \mathrm{nM}$ of SR95531 (Tocris Bioscience, Bristol, UK), antagonist of the neuroinhibitor $\mathrm{GABA}_{A}$, was added to the aCSF. SR95531 lowers the activation barrier for neuronal firing making the callosal system hyperexcitable to gain a measureable signal to stimulation. To stimulate, we used inbath tungsten electrodes that did not directly contact the slice. Electrical pulse train stimulations were applied every $10 \mathrm{~s}$ for 50 repetitions in all experiments. Each stimulation is a train of $2 \mathrm{~ms}$ pulses at $40 \mathrm{~Hz}$ for $1 \mathrm{~s}$. Amplitudes varied from 1 to $6 \mathrm{~mA}$ to achieve an initial excitability of around $10 \%$ in every experiment. Excitability is defined as the number of FA responses over the number of stimulations.

\section{Strain Field Calculation}

Strain could be defined with different reference points. Here, we provided measurements of two strain values. The global strain was calculated based on the displacement of the micro-vices alone. It is the distance traveled by the moving micro-vice over the original separation between the 2 micro-vices.
We also measured the strain field in the region with FA responses using digital image correlation (DIC) analysis (Jones et al., 2014). We called this the local strain. Briefly, every image (except the first one) in an image sequence was divided into small subregions. Each subregion was treated as rigid (no deformation whatsoever). These subregions were then compared to the first image in the sequence to find the location of maximum correlation. This way a displacement vector could be formulated for each subregion in every image. The displacement vectors could then be interpolated and smoothed to give a numerical displacement field. The displacement values (u) were then formulated into a 2-D strain tensor field (Figure 1D), given the plane stress assumption, using the following strain-displacement relationship:

$$
\epsilon_{\alpha \beta}=\frac{1}{2}\left(\frac{\partial u_{\alpha}}{\partial X_{\beta}}+\frac{\partial u_{\beta}}{\partial X_{\alpha}}\right),
$$

where $\mathrm{X}$ is the coordinate system, and $\alpha \beta=1,2$ define the inplane coordinates. When the 2 indices are the same, i.e., $\alpha=$ $\beta=1$ or 2 , it is denoting the axial strains. When the 2 indices are different, i.e., $\alpha \neq \beta$, it is the shear component induced by the inhomogeneity of the brain slice. To summarize the strain field, essentially a 2-by-2 tensor, we adopted the effective strain convention. Effective strain $\left(\epsilon_{\mathrm{e}}\right)$, reported as local strain in text, can be defined as:

$$
\epsilon_{e}=\sum_{\beta=1}^{2} \sum_{\alpha=1}^{2} \sqrt{\frac{2}{3} \epsilon_{\alpha \beta} \epsilon_{\alpha \beta}} .
$$


The local effective strain serves to account for also the shear components in the local region, whereas the global strain has only axial components. Global strain is what is applied, while local strain is the resulting strain level in the region of interest.

\section{Quantification of Evoked Activation}

A criterion is required to identify the active pixels for an accurate representation of the spatial and temporal FA activation. We used the coefficient of variation, $I_{c v}$, (standard deviation over mean) of temporal intensities of each pixel to find the active pixels in our image sequences. To determine the threshold for quantification of activation area, we plotted the $I_{c v}$ distribution into a histogram, $H\left[I_{c v}\right]$, and fit the profile to a two-term Gaussian function, $G\left(I_{c v}\right)$. The selection of two-term Gaussian was because all images could be segmented into a darker region and a brighter region. The Gaussian distribution could then capture random noises fluctuating around the respective mean. The right tail (with larger values) is expected to deviate from the normal distribution because of the fluorescing pixels, and we use this point of deviation as our $I_{c v}$ threshold value (Figure 1E). The point of deviation, $I_{c v}^{p}$, is defined as:

$$
I_{c v}^{p}: I_{c v} \rightarrow \frac{H-G}{G}>0.5
$$

It is essentially when the actual distribution $(\mathrm{H})$ exceeds the Gaussian (G) by $50 \%$. To quantify the time profile of activation of the activated region (Figure 1F), spatial averaging of intensity was used. The change in activation is quantified by $\frac{\Delta f}{f_{0}}$, defined as the change in fluorescence over baseline fluorescence.

During the measurement of spontaneous activities, the slice was constantly moving. This led to a problem of uneven lighting as we progressed through the image sequence, affecting the FA signal. In an effort to correct this artifact, we established and reported the adjusted intensity. The adjusted intensity $I_{a d j}$ is defined as:

$$
\begin{aligned}
I_{a d j}[t]= & \sum_{x_{i}, y_{i} \in A}^{N} \frac{I\left[x_{i}, y_{i}, t\right]}{N} \\
& -\left(\sum_{x_{i}=x_{\min }}^{x_{\max }} \sum_{y_{i}=y_{\min }}^{y_{\max }} \frac{I\left[x_{i}, y_{i}, t\right]}{\Delta x \Delta y-N}-\sum_{x_{i}, y_{i} \in A}^{N} \frac{I\left[x_{i}, y_{i}, t\right]}{\Delta x \Delta y-N}\right),
\end{aligned}
$$

where A denotes the activation area. $x_{\max }, x_{\min }, y_{\max }$, and $y_{\min }$ are the limit points of the smallest rectangle enclosing the entire activation area. $\Delta x=x_{\max }-x_{\min } . \Delta y=y_{\max }-y_{\min }$. This could be interpreted as the signal minus the average background intensity. The first term in Equation (4) denotes the signal averaged over the activation area. The second term denotes averaged background intensity.

\section{Results}

\section{Flavoprotein Autofluorescence before Stretch and after Stretch}

Using the platform we developed (Figure 1A), the brain slice was gently gripped and tensed by the micro-vices until no edge slippage was observed ensuring that the prescribed stretch was entirely applied onto the slice. (see Figure 1B and Materials and Methods for detailed description). As baseline measurement, we took a fluorescence video of $500 \mathrm{~s}$ long recording the response of the slice to 50 stimulation pulse trains, each $10 \mathrm{~s}$ apart. The slice was subsequently stretched by $317.5 \mu \mathrm{m}$ (or $4.2 \%$ of total the length of the slice) over $4 \mathrm{~s}$ (Figure 1C). Using the DIC methods (see Materials and Methods), effective strain at responsive area was found to be $2.5 \%$ (Figure 1D), while edge-to-edge global strain is $4.2 \%$. The stretch was maintained for $10 \mathrm{~min}$ and then entirely released. No measurement was made while the slice was being held stretched. After the slice was released from stretch, we performed the baseline measurement again. This stretchbaseline-cycle was repeated for 4 more times. This paradigm was applied to 4 independent slices from 4 animals.

To ensure that the extra excitability is not due to sensitization to electrical stimulations or degeneration of tissue health, control slices were subjected to the same series of manipulation, without the stretch. The control paradigm was applied to 3 independent slices from 3 animals.

\section{Stretch Effects on Excitability}

Excitability here is defined as the ratio of number of times the slice responds (detected using FA) to the number it is electrically stimulated. Thus, excitability gives the probability of FA response. We found that in all slices undergoing the stretch-baseline-cycle $(n=4)$, excitability increases after every cycle to ultimately 3 times of the original probability (Figure 2A). It thus seems that the slice "remembers" its past history of stretch, and its current excitability results from a cumulative effect of its past stretches. Normalized baseline measurements from the control group $(n=$ 3 ) are plotted next to stretched group. We fit a linear regression to each experiment individually, and the averaged slope is reported in Figure 2B comparing the slope in stretched and control slices. We found that the stretched slices showed a significant increase in excitability compared to control slices $(p=0.013)$.

\section{Stretch Effects on Spatial and Temporal Activation}

We note that the area of activation of the slice fluctuates in the stretched group (Figure 3A). Further looking into the spatial distribution and the strength of variation in the activation area, we observed a shift in the location of maximum activity in the stretched group. An example is shown in Figure 3A, with the stretched group at the top row and the control group at the bottom. It is thus possible that the stretch applied and released in between the 2 baseline measurements can lead to a spatial reconfiguration of excitability.

Temporal activation profile, $\frac{\Delta f}{f_{0}}$, is plotted against time. The amplitude of each fluorescence peak is obtained by subtracting the value preceding the stimulation to the peak $\frac{\Delta f}{f_{0}}$ value. The first activation amplitude, i.e., the first electrical stimulation after the stretch is released, in each baseline measurement is found to be steady (Figure 3B). However, the average activation amplitude is found to decrease after each stretch-baseline cycle. The control amplitudes remain unchanged (Figure 3C). We fit a linear regression to the amplitudes in each experiment individually, and 


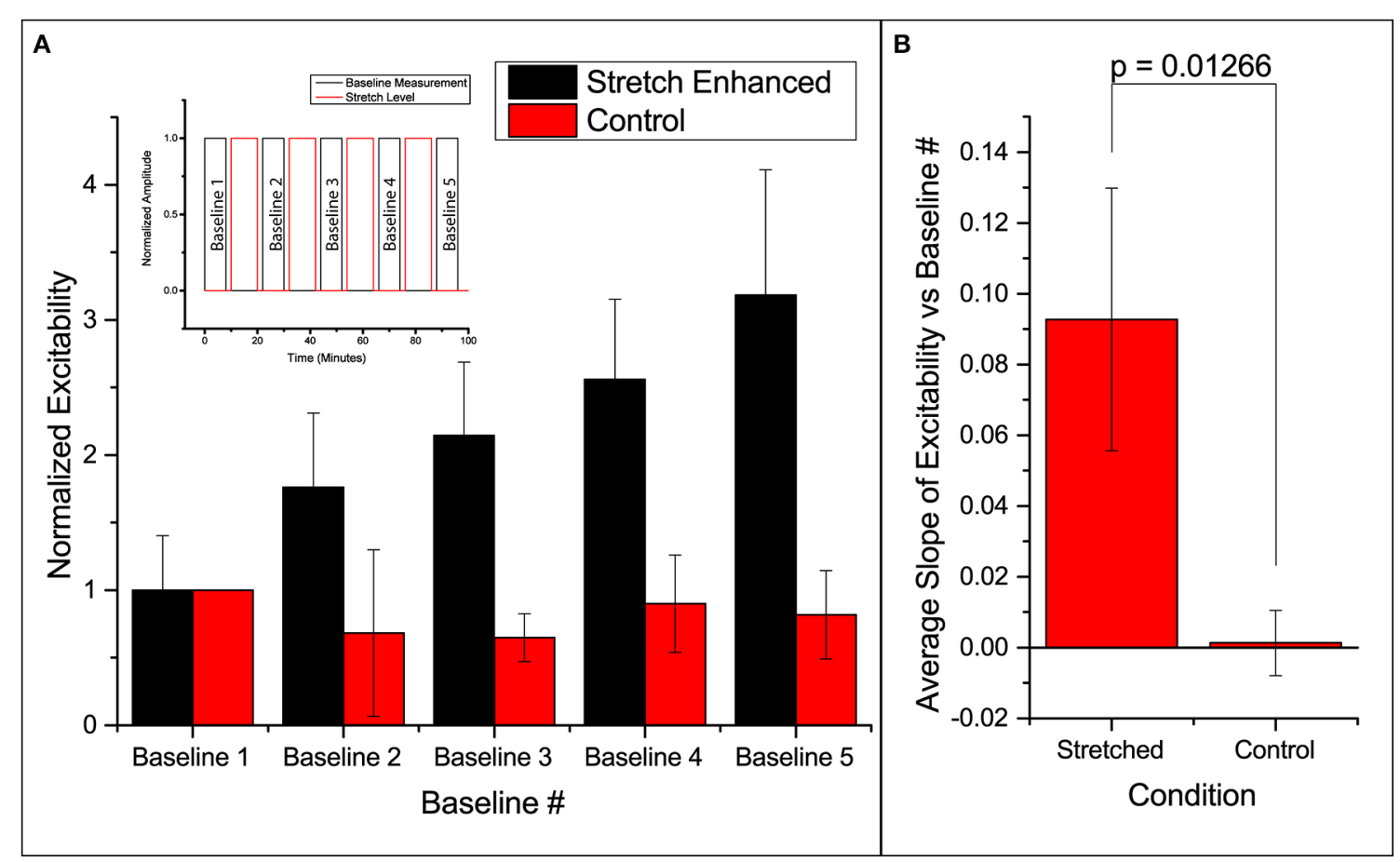

FIGURE 2 | Enhanced excitability of callosal pathway by stretch alone and the 2 control schemes. (A) Normalized probability of response of stretched group and control group. The stretched data set here is individually normalized to the average probability of the first baseline. This serves to show the variation in the starting probability of response, as given in the error bar. All subsequent column plots are normalized to the first baseline probability within individual experimental data set, leading to an error bar magnitude of 0 . Subfigure includes a schematic of the paradigm. (B) The slopes of excitability vs. baseline \# in each independent data set are averaged and reported here. This serves to compare the increase in excitability of the stretched and control groups. All error bars in SD. $P$-value obtained from 2 -tail $t$-test with unequal variance. the averaged slope is reported in Figure 3D. All stretched slices show significant negative slope compared to controls $(p=0.041)$ and a significant negative slope of the temporal activation rate $(p=0.028$, Figure 3E). The temporal activation rate serves as a secondary measurement of the temporal activation. It is equivalent to the maximum rate $\frac{d\left(\frac{\Delta f}{f_{0}}\right)}{d t}$ when the FA signal is ascending to the peak value (FA amplitude).

The attenuations in the signal appear to correlate with the number of FA responses. This leads to the question whether the attenuation of activation amplitude and rate is simply due to the increase in the number of FA responses or is actually induced by the stretching paradigm. To address this question, we artificially increased the probability of FA response by increasing the applied electrical stimulation $(n=4)$. A linear regression is fit to the amplitude/rate vs. excitability profile in individual experiment separately (Figures 3F,G). The average slope (slightly positive but approaching zero) shows that the activation amplitude and rate do not change with electrical stimulation alone. This is possibly because at subthreshold stimulations, increasing electrical current does not lead to an FA response with a larger amplitude. The extra energy is exhausted by the higher number of FA occurrences. Thus, we conclude that the attenuation of the signals is due to the stretching paradigm rather than a consequence of electrical stimulation, suggesting that the stretching manipulation might have lowered the energy barrier for synaptic activation leading to hyperexcitability.

\section{Spontaneous Activities due to Stretch Alone}

By introducing a higher concentration of SR95531, it would lead to spontaneous FA activities previously not observed. A separate slice was stretched with time (Figure 4A) in a stepwise fashion in 3 cycles with a higher dose of SR95531 (2 $\mu \mathrm{M})$. There was a 5min time gap between each cycle. No electrical stimulation was applied here. The slice was imaged during the entire span of the cycles and FA data were analyzed as described in Equation (4) (see Materials and Methods).

The $\frac{\Delta f}{f_{0}}$ plots show a close correspondence between stretching impulse (strain rate $>0$ ) and induced flavoprotein activities in all three cycles of stretch (Figure 4A). Some spontaneous FA signals could be observed when the slice was not stretched or when simply held stretched, i.e., strain rate $=0$. No activity was observed during the relaxation steps (strain rate $<0$ ). Out of the 11 stretching steps, 6 (50\%) led to an immediate FA response; two others led to FA responses within $13 \mathrm{~s}$ (Figure 4B). The results suggest that a brain slice is sensitive to also stretch impulses.

\section{Discussion}

The probability of neural response to electrical stimulation can be increased by applying a stronger electrical stimulus or by 


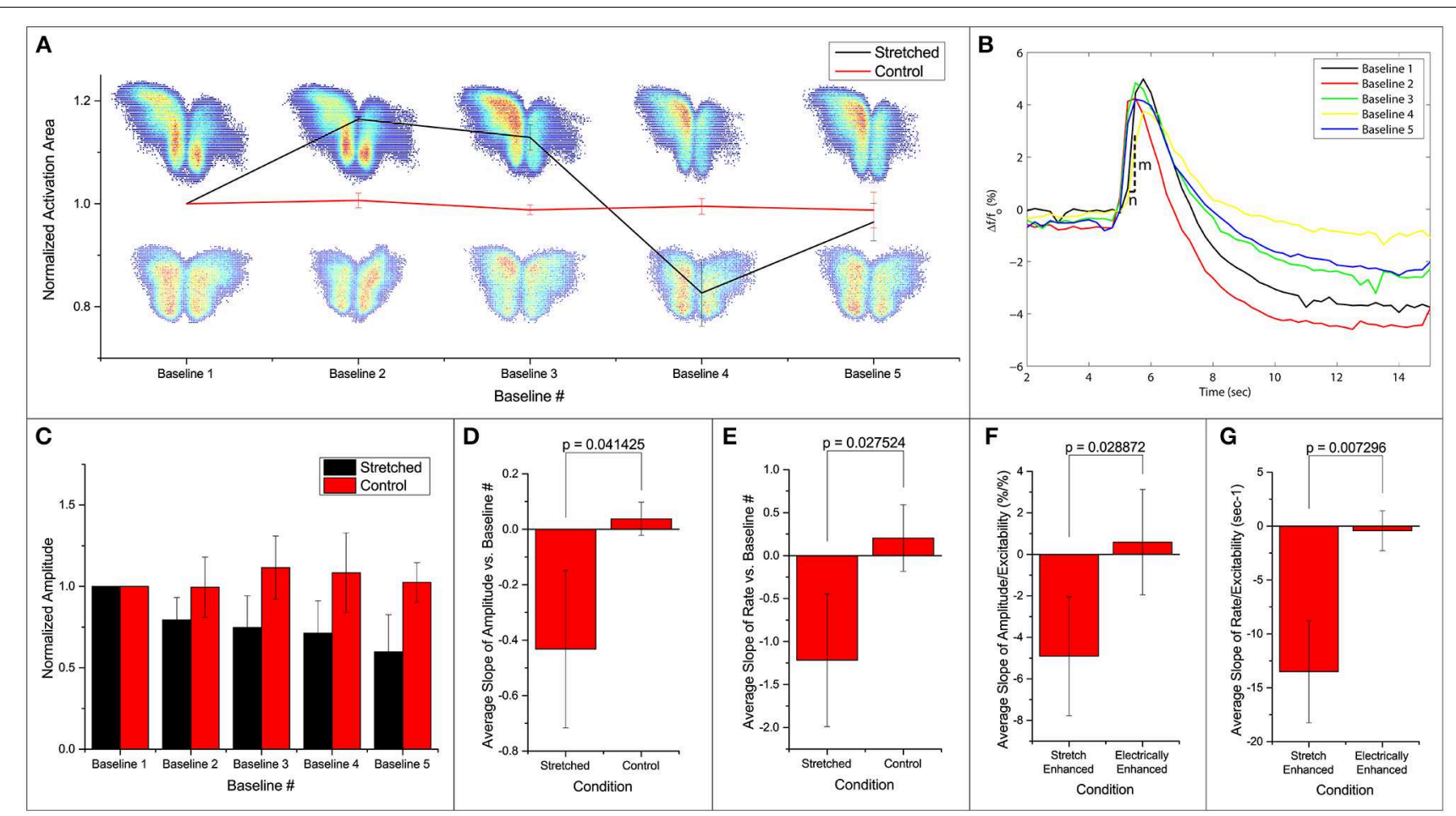

FIGURE 3 | Fluorescence intensities are averaged over the calculated activation areas. (A) Spatial activities in subsequent baseline measurements. Normalized activation area of the stretched and control groups are plotted. Two examples of activation areas: stretched on top, and control at the bottom. (B) An example of the $\frac{\Delta f}{f_{0}}$ signal in one stretch experiment. Each line denotes the first FA response (out of 50 stimulations) in baseline $1-5$ as labeled. All measurements are made when stretch is zero.
After each baseline measurement, slice is held stretched for 10 min and then brought back to the original position before the next baseline measurement commences. Activation rate is defined as $\frac{m}{n}$. (C) Normalized average amplitudes are plotted for the stretched and control groups. Slope of (D,F) amplitude and $\mathbf{( E , G ) ~ r a t e ~ v s . ~ b a s e l i n e ~ m e a s u r e m e n t ~ n u m b e r ~ a n d ~ e x c i t a b i l i t y ~}$ from each experiment is averaged and compared. All error bars in SD. $P$-values obtained from 2 -tail t-test with unequal variance. applying the stimulus more frequently (Bikson et al., 2004). Long-term potentiation (LTP) has been shown to be induced by a tetanic stimulation in which frequency is usually in the range of $100 \mathrm{~Hz}$ (Malenka and Bear, 2004). Long-term depression (LTD), on the other hand, can be induced by a low frequency stimulation in the range of $10 \mathrm{~Hz}$ (Malenka and Bear, 2004). The actual frequency that is required to induce plasticity is pathwaydependent and species-dependent (Malenka and Bear, 2004). At intermediate frequencies, synaptic transmission is usually memoryless, i.e., the same electrical stimulus will give the same probability/strength of neural responses regardless of what the pathway was subjected to before (Bliss and Lomo, 1973).

Here we show that by maintaining a small stretch for $10 \mathrm{~min}$, we can increase this probability of neural response. Note that this is not the same as LTP, since LTP describes the changes in strength of postsynaptic response, whereas we are reporting excitability. Nonetheless, in both LTP and the stretch-enhanced excitability, the electrically evoked activity has a higher probability in surpassing the activation threshold.

It is not yet known whether the changes in probability, amplitude or rate of the FA response correlate with similar change in the underlying electrophysiological response. However, it has been shown that there is a strong correspondence in the strength of the 2 signals under normal conditions (Shibuki et al., 2003; Reinert et al., 2004; Llano et al., 2009). It has also been shown that only $15 \%$ of the FA signal is left when postsynaptic glutamate receptors are blocked (Reinert et al., 2004; Llano et al., 2012). Therefore, it is likely the increase in excitability we see here are due to direct/indirect enhancement in postsynaptic activity-either as a result of more vesicles being released from the presynaptic terminal upon the same stimulation, or the mechanism of receiving the vesicles has become more efficient.

Previous finding reports that a $5 \%$ stretch in embryonic Drosophila axons of motor neurons results in a $200 \%$ increase in neurotransmitter vesicle clustering at the presynaptic terminal of neuromuscular junctions. It takes about $20 \mathrm{~min}$ for the increased clustering to occur (Siechen et al., 2009; Ahmed et al., 2012). In our case, the stretch was held for $10 \mathrm{~min}$. Arguably, the difference could be attributed to the different species and the different neural structures (neuromuscular vs. neural-neural). It is thus possible that the increased clustering of synaptic vesicles can lead to a higher docking ratio, more frequent spontaneous release, and larger release upon stimulation (Dobrunz, 2002). This could explain the higher excitability upon completion of a stretch-baseline cycle. 


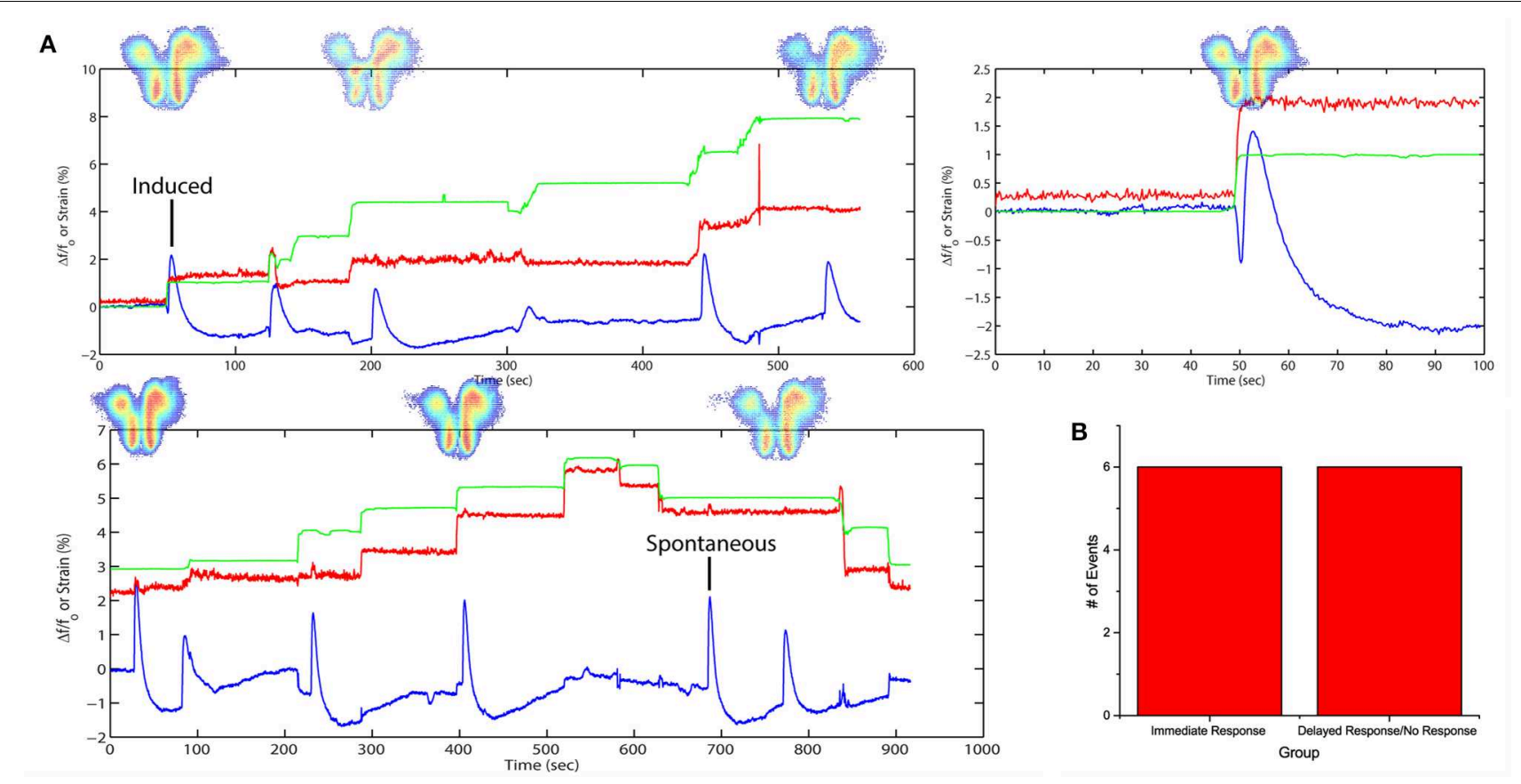

FIGURE 4 | Stretch effects alone without electrical stimulation. (A) Processed average intensities are plotted against time. Red lines denote effective strain level at activation area. Green lines denote effective strain level globally, defined by the position of the micro-vices. Activation area examples are placed aligned to respective peaks. An example is given for induced and spontaneous activation respectively. (B) All fluorescence occurrences are grouped into 2 groups: (1) immediate firing after stretch application, (2) delayed firing or no firing before the next stretch.
The increased response due to stretch could be because of postsynaptic modifications, in particular, that stretch results in increased clustering of neurotransmitter receptors (AMPA and/or NMDA) in the postsynaptic density, similar to LTP (Bear et al., 1987). This increases the probability of neuronal activity due to a given electrical stimulation and hence a given presynaptic release of neurotransmitter. It has been shown that integrin activities (to be discussed) lead to phosphorylation of CamKII (Charrier et al., 2010), known to be critical in LTP (Lisman et al., 2012).

Cell-cell adhesion protein molecules facilitate force transfer in neighboring cells by acting as an anchor (Wang et al., 2009). Many of these protein complexes are found in neural pathways (Mobley et al., 2009). Increased mechanical tension can lead to a structural change in these molecules leading to downstream signaling cascades. One study has attributed the high stretch sensitivity of motor nerve terminals to integrin, a cell-cell adhesion protein (Chen and Grinnell, 1995). In that study, both the evoked amplitude and the frequency of spontaneous activity increase significantly with the application of stretch on frog muscle. The increase is linear with respect to the magnitude of the stretch applied. The known integrin inhibitors, such as peptides containing the Arg-Gly-Asp (RGD) sequence, suppressed the stretch sensitivity. Enhancement of transmitter release occurs within a few milliseconds of the stretch application, which might affect the stretch-rate sensitivity of neuronal response, i.e., increased responsiveness at faster rate of stretch.

Stretch or tension can also increase ionic conductances, similar to peripheral mechanotransducers (Sachs, 1991), either pre- or post-synaptically. Collectively, or individually, alterations in ionic conductances could lower the activation barrier for action potentials or increase postsynaptic sensitivity to presynaptic activity. The change of conductance due to stretch is expected to be instantaneous. Thus, this mechanism should also result in strain-rate sensitivity, possibly providing an explanation for the similar sensitivity we observed.

There are also clinical implications to this work. Stretching of axons is seen in a large number of clinical conditions, including hydrocephalus, traumatic brain injury and other forms of brain injury leading to edema (e.g., stroke, tumors, infection). The current results imply that even small amounts of stretch can lead to major changes in neuronal function, and may explain hyperexcitability phenomena, such as seizures, that can be seen during these states. We note that some studies have shown that traumatic levels of stretch typically lead to hypoexcitability (Goforth et al., 2011; Ferrario et al., 2013). It is thus possible that different strength of mechanical stimuli can lead to opposite outcomes. Future work will clarify the mechanisms of the changes we observed, and may therefore lead to novel therapeutics to deal with these stretch-induced hyperexcitability. 


\section{Author Contributions}

AF, KS, DL, TS designed research. AF, KS performed research. AF contributed unpublished reagents/analytic tools. AF, TS analyzed data. AF, TS wrote the paper. All authors read and reviewed the paper.

\section{References}

Ahmed, W. W., Li, T. C., Rubakhin, S. S., Chiba, A., Sweedler, J. V., and Saif, T. A. (2012). Mechanical tension modulates local and global vesicle dynamics in neurons. Cell. Mol. Bioeng. 5, 155-164. doi: 10.1007/s12195-012-0223-1

Bear, M. F., Cooper, L. N., and Ebner, F. F. (1987). A physiological basis for a theory of synapse modification. Science 237, 42-48. doi: 10.1126/science.30 37696

Morrison, B. III., Elkin, B. S., Doll'e, J. P., and Yarmush, M. L. (2011). In vitro models of traumatic brain injury. Annu. Rev. Biomed. Eng. 13, 91-126. doi: 10.1146/annurev-bioeng-071910-124706

Bikson, M., Inoue, M., Akiyama, H., Deans, J. K., Fox, J. E., Miyakawa, H., et al. (2004). Effects of uniform extracellular DC electric fields on excitability in rat hippocampal slices in vitro. J. Physiol. 557, 175-190. doi: 10.1113/jphysiol.2003.055772

Bliss, T. V., and Lomo, T. (1973). Long-lasting potentiation of synaptic transmission in the dentate area of the anaesthetized rabbit following stimulation of the perforant path. J. Physiol. 232, 331-356. doi: 10.1113/jphysiol.1973.sp010273

Charrier, C., Machado, P., Tweedie-Cullen, R. Y., Rutishauser, D., Mansuy, I. M., and Triller, A. (2010). A crosstalk between $\beta 1$ and $\beta 3$ integrins controls glycine receptor and gephyrin trafficking at synapses. Nat. Neurosci. 13, 1388-1395. doi: $10.1038 / \mathrm{nn} .2645$

Chen, B. M., and Grinnell, A. D. (1995). Integrins and modulation of transmitter release from motor nerve terminals by stretch. Science 269, 1578-1580. doi: $10.1126 /$ science. 7667637

Cohen, A. S., Pfister, B. J., Schwarzbach, E., Grady, M. S., Goforth, P. B., and Satin, L. S. (2007). Injury-induced alterations in CNS electrophysiology. Prog. Brain Res. 161, 143-169. doi: 10.1016/S0079-6123(06)61010-8

Dobrunz, L. E. (2002). Release probability is regulated by the size of the readily releasable vesicle pool at excitatory synapses in hippocampus. Int. J. Dev. Neurosci. 20, 225-236. doi: 10.1016/S0736-5748(02)00015-1

Ferrario, C. R., Ndukwe, B. O., Ren, J., Satin, L. S., and Goforth, P. B. (2013). Stretch injury selectively enhances extrasynaptic, GluN2B-containing NMDA receptor function in cortical neurons. J. Neurophysiol. 110, 131-140. doi: $10.1152 /$ jn. 01011.2012

Franze, K., Janmey, P. A., and Guck, J. (2013). Mechanics in neuronal development and repair. Annu. Rev. Biomed. Eng. 15, 227-251. doi: 10.1146/annurev-bioeng071811-150045

Goforth, P. B., Ren, J., Schwartz, B. S., and Satin, L. S. (2011). Excitatory synaptic transmission and network activity are depressed following mechanical injury in cortical neurons. J. Neurophysiol. 105, 2350-2363. doi: 10.1152/jn.00467.2010

Jones, E. M. C., Silberstein, M. N., White, S. R., and Sottos, N. R. (2014). In situ measurements of strains in composite battery electrodes during electrochemical cycling. Exp. Mech. 54, 971-985. doi: 10.1007/s11340-0149873-3

Kandel, E., Schwartz, J., Jessell, T., Siegelbaum, S., and Hudspeth, A. J. (2012). Principles of Neural Science, 5th Edn. New York, NY: McGraw Hill Professional.

Lajtha, A., Gibson, G. E., and Dienel, G. A. (2007). Handbook of Neurochemistry and Molecular Neurobiology. New York, NY: Springer.

Lamoureux, P., Ruthel, G., Buxbaum, R. E., and Heidemann, S. R. (2002). Mechanical tension can specify axonal fate in hippocampal neurons. J. Cell Biol. 159, 499-508. doi: 10.1083/jcb.200207174

Lisman, J., Yasuda, R., and Raghavachari, S. (2012). Mechanisms of CaMKII action in long-term potentiation. Nat. Rev. Neurosci. 13, 169-182. doi: $10.1038 / \mathrm{nrn} 3192$

\section{Acknowledgments}

This work was funded by the National Science Foundation (NSF 1300808) Grant 0965918 IGERT at UIUC: Training the Next Generation of Researchers in Cellular and Molecular Mechanics and Bio-Nanotechnology.

Llano, D. A., Slater, B. J., Lesicko, A. M., and Stebbings, K. A. (2014). An auditory colliculothalamocortical brain slice preparation in mouse. J. Neurophysiol. 111, 197-207. doi: 10.1152/jn.00605.2013

Llano, D. A., Theyel, B. B., Mallik, A. K., Sherman, S. M., and Issa, N. P. (2009). Rapid and sensitive mapping of long-range connections in vitro using flavoprotein autofluorescence imaging combined with laser photostimulation. J. Neurophysiol. 101, 3325-3340. doi: 10.1152/jn.91291. 2008

Llano, D. A., Turner, J., and Caspary, D. M. (2012). Diminished cortical inhibition in an aging mouse model of chronic tinnitus. J. Neurosci. 32, 16141-16148. doi: 10.1523/JNEUROSCI.2499-12.2012

Malenka, R. C., and Bear, M. F. (2004). LTP and LTD: an embarrassment of riches. Neuron 44, 5-21. doi: 10.1016/j.neuron.2004.09.012

Mobley, A. K., Tchaicha, J. H., Shin, J., Hossain, M. G., and McCarty, J. H. (2009). Beta8 integrin regulates neurogenesis and neurovascular homeostasis in the adult brain. J. Cell Sci. 122, 1842-1851. doi: 10.1242/jcs. 043257

Morris, C. E. (2011). Voltage-gated channel mechanosensitivity: fact or friction? Front. Physiol. 2:25. doi: 10.3389/fphys.2011.00025

Patel, T. P., Ventre, S. C., Geddes-Klein, D., Singh, P. K., and Meaney, D. F. (2014). Single-neuron NMDA receptor phenotype influences neuronal rewiring and reintegration following traumatic injury. J. Neurosci. 34, 4200-4213. doi: 10.1523/JNEUROSCI.4172-13.2014

Pfister, B. J., Bonislawski, D. P., Smith, D. H., and Cohen, A. S. (2006). Stretchgrown axons retain the ability to transmit active electrical signals. FEBS Lett. 580, 3525-3531. doi: 10.1016/j.febslet.2006.05.030

Pfister, B. J., Iwata, A., Meaney, D. F., and Smith, D. H. (2004). Extreme stretch growth of integrated axons. J. Neurosci. 24, 7978-7983. doi: 10.1523/JNEUROSCI.1974-04.2004

Reinert, K. C., Dunbar, R. L., Gao, W., Chen, G., and Ebner, T. J. (2004). Flavoprotein autofluorescence imaging of neuronal activation in the cerebellar cortex in vivo. J. Neurophysiol. 92, 199-211. doi: 10.1152/jn.01275. 2003

Reinert, K. C., Gao, W., Chen, G., and Ebner, T. J. (2007). Flavoprotein autofluorescence imaging in the cerebellar cortex in vivo. J. Neurosci. Res. 85, 3221-3232. doi: 10.1002/jnr.21348

Sachs, F. (1991). Mechanical transduction by membrane ion channels: a mini review. Mol. Cell. Biochem. 104, 57-60. doi: 10.1007/BF002 29804

Sachs, F. (2010). Stretch-activated ion channels: what are they? Physiology 25, 50-56. doi: 10.1152/physiol.00042.2009

Santhakumar, V., Ratzliff, A. D., Jeng, J., Toth, Z., and Soltesz, I. (2001). Long-term hyperexcitability in the hippocampus after experimental head trauma. Ann. Neurol. 50, 708-717. doi: 10.1002/ana.1230

Shibuki, K., Hishida, R., Murakami, H., Kudoh, M., Kawaguchi, T., Watanabe, M., et al. (2003). Dynamic imaging of somatosensory cortical activity in the rat visualized by flavoprotein autofluorescence. J. Physiol. 549, 919-927. doi: 10.1113/jphysiol.2003.040709

Siechen, S., Yang, S., Chiba, A., and Saif, T. (2009). Mechanical tension contributes to clustering of neurotransmitter vesicles at presynaptic terminals. Proc. Natl. Acad. Sci. U.S.A. 106, 12611-12616. doi: 10.1073/pnas.09018 67106

Smith, D. H., Wolf, J. A., and Meaney, D. F. (2001). A new strategy to produce sustained growth of central nervous system axons: continuous mechanical tension. Tissue Eng. 7, 131-139. doi: 10.1089/1076327013000 62714 
Theyel, B. B., Llano, D. A., Issa, N. P., Mallik, A. K., and Sherman, S. M. (2011). In vitro imaging using laser photostimulation with flavoprotein autofluorescence. Nat. Protoc. 6, 502-508. doi: 10.1038/nprot.2011.315

Tyler, W. J. (2012). The mechanobiology of brain function. Nat. Revie. Neurosci. 13, 867-878. doi: $10.1038 / \mathrm{nrn} 3383$

Van Essen, D. C. (1997). A tension-based theory of morphogenesis and compact wiring in the central nervous system. Nature 385, 313-318. doi: $10.1038 / 385313 \mathrm{a} 0$

Wang, N., Tytell, J. D., and Ingber, D. E. (2009). Mechanotransduction at a distance: mechanically coupling the extracellular matrix with the nucleus. Nat. Rev. Mole. Cell Biol. 10, 75-82. doi: 10.1038/nrm2594
Conflict of Interest Statement: The authors declare that the research was conducted in the absence of any commercial or financial relationships that could be construed as a potential conflict of interest.

Copyright (c) 2015 Fan, Stebbings, Llano and Saif. This is an open-access article distributed under the terms of the Creative Commons Attribution License (CC $B Y)$. The use, distribution or reproduction in other forums is permitted, provided the original author(s) or licensor are credited and that the original publication in this journal is cited, in accordance with accepted academic practice. No use, distribution or reproduction is permitted which does not comply with these terms. 